\title{
COVID-19 and pregnancy: the fruitful experience of the university hospital of Tangier Morocco with hydroxychloroquine and anti- retroviral treatment
}

\author{
Karima Aouali*, Houda Moustaid, Saad Benkirane
}

Department of Gynaecology and Obstetrics, Tangier Tetouan Al hoceima University and Hospital, Tangier, Morocco

\author{
Received: 22 November 2020 \\ Revised: 14 January 2021 \\ Accepted: 15 January 2021 \\ *Correspondence: \\ Dr. Kaima Aouali, \\ E-mail: aouali.karima1@gmail.com
}

Copyright: (c) the author(s), publisher and licensee Medip Academy. This is an open-access article distributed under the terms of the Creative Commons Attribution Non-Commercial License, which permits unrestricted non-commercial use, distribution, and reproduction in any medium, provided the original work is properly cited.

\begin{abstract}
The coronavirus disease 2019 (COVID-19) caused by the emerging virus, severe acute respiratory distress syndrome coronavirus 2 (SARS-CoV-2), was announced as a pandemic by the World Health Organization as a global public health emergency. Since the first case of COVID-19 was declared the Moroccan government set up drastic preventative measures to deal with the spread of the virus such as compulsory containment, telework, distance education, use of masks in public places, and many other preventive measures. Pregnant women require special attention in relation to prevention, diagnosis and management. We herein reported a prospective observational study that aims to evaluate the security and effectiveness of hydroxychloroquine on pregnant women with corona virus disease. Our study is a prospective study performed in the three hospitals specialized on corona virus disease 2019 in tangier morocco. We included pregnant women hospitalized with COVID-19 and reproductive-aged. Most cases took the hydroxychloroquine treatment. The maternal outcomes under hydroxychloroquine appear good in most cases for both mother and child and seemed to be safe according to several studies on its use for rheumatic diseases. In the absence of more robust data, this treatment might be best practice in countries that can't afford the expensive antiviral treatment in this outbreak especially in emergency situations.
\end{abstract}

Keywords: Pregnancy, Corona virus 2019, Hydroxychloroquine

\section{INTRODUCTION}

The coronavirus disease 2019 (COVID-19) caused by the emerging virus, severe acute respiratory distress syndrome coronavirus 2 (SARS-CoV-2), was announced as a pandemic by the World Health Organization is a global public health emergency. ${ }^{1}$

Since the first case of COVID-19 was declared the Moroccan government set up drastic preventative measures to deal with the spread of the virus such as compulsory containment, telework, distance education, use of masks in public places, and many other preventive measures.

Pregnant women have their special immunological adaptation. $^{2}$ The state of the transient suppressed immunity is modulated by suppressing $\mathrm{T}$ cells activities, and hence predisposes pregnant women to virus infection. ${ }^{3}$ Pregnant women require special attention in relation to prevention, diagnosis and management. we herein reported a prospective observational study that aims to evaluate the security and effectiveness of hydroxychloroquine on pregnant women with corona virus disease. 


\section{CASE SERIES}

Prospective study performed in the three hospitals specialized on corona virus disease 2019 in tangier morocco. We included pregnant women hospitalized with COVID-19 and reproductive-aged.

The therapeutic choice according to the recommendations of the health ministry was made of 1) azithromycin :500mg for the first day then $250 \mathrm{mg}$ from day 2 to day 7

2) vitamin $C: 1 \mathrm{~g}$ three times a day for 10 days 3 ) vitamin D: 100000 UI every two weeks for 2 months 4) zinc sulfate: $125 \mathrm{mg}$ per day for 5 days.

In the absence of any clinical improvement for the next 5 days of treatment, and after patient's consent, lopinavir $800 \mathrm{mg}$ per day or hydroxychloroquine $600 \mathrm{mg}$ per day for 10 days were prescribed.

Systematic throboprophylaxis was administered for all our patients for the 15days of their hospital stay.

A patient is declared as a COVID-19 free after two consecutive negative test results.

\section{Case 1}

B. L a 21-year-old primigest, at her 41th week of pregnancy patient had consulted for fever, myalgia, asthenia and headache without any respiratory symptoms then tested positive to covid19, the obstetric ultrasound showed a healthy baby with and estimated fetal weight of $3 \mathrm{~kg} 600$ with amniotic fluid in normal amount. She benefited from a cesarean for clinically suspect pelvis and gave birth to a negative COVID-19 new born then she was put on hydroxychloroquine associated to azithromycin treatment, declared cured and asymptomatic after two weeks.

\section{Case 2}

B. $\mathrm{N}$ a 25-year-old at her 23th weeks of pregnancy with no medical background, who presented with cough, dyspnea and dizziness the COVID-19 test was positive for fetal condition the obstetric ultrasound was without anomalies, fetal heart-rate (FHR) monitoring was normal as well, after 5 days of no clinical improvement and after consent the patient received hydroxychloroquine treatment became asymptomatic at the end of the fourth day of treatment, no perinatal complications were observed, then she tested negative after 10 days.

\section{Case 3}

S. G a 28-year-old at her 29th week of pregnancy, without any medical background had consulted for digestive signs: nausea, vomiting, and diarrhea associated to fever, the obstetric ultrasound was normal, fetal heartrate (FHR) monitoring was normal as well, after 5 days of no clinical improvement, and after consent she was put on hydroxychloroquine drug then two days later on lopinavir at her request, the patient is no longer symptomatic but still testing positive to COVID-19 at her 20th day of treatment.

\section{Case 4}

B. S a 23-year-old at her 29th week of pregnancy, without any medical background had consulted for simple anosmia then tested positive for COVID-19 the obstetric ultrasound was normal, in addition to a normal fetal heart-rate (FHR) monitoring, after 5 days of no clinical improvement, then after 20 days of lopinavir treatment following her personal choice, she's always positive to COVID-19, without any fetal complications.

\section{Case 5}

B. B a 20-year-old at her 39th week of pregnancy, with a scared uterus for a breech presentation two years ago had consulted for digestive signs: nausea, vomiting, associated to fever, the obstetric ultrasound showed a breech presentation, fetal heart-rate (FHR) monitoring was normal too, a C-section was realized for breech presentation on a scared uterus, after 5 days of no clinical improvement, and after consent she was put on hydroxychloroquine drug and declared cured at her 15th day of treatment with the disappearance of all symptoms, and the baby was always testing negative to COVID-19.

\section{Case 6}

B. M a 41-year-old at her 40th week of pregnancy, with a scared uterus for A fetal macrosomia one year ago had consulted for asthenia and fever, the obstetric ultrasound showed a macrosomia, fetal heart-rate (FHR) monitoring was also normal, a C-section was realized for macrosomia on a scared uterus, the baby tested negative to COVID-19 with a birth weight of $4 \mathrm{~kg} 500$, after 5 days of no clinical improvement and after consent she was put on hydroxychloroquine drug and declared cured and was symptoms free at her 12th day of treatment.

\section{Case 7}

A. N a 22-year-old at her 34th week of pregnancy, without any medical background, had consulted for asthenia and fever, the obstetric ultrasound was normal, along with the fetal heart-rate (FHR) monitoring which was normal, she was put on the first-choice treatment, after 5 days she was no longer symptomatic, then tested negative by the end of 10 days.

\section{Case 8}

B. L a 28-year-old at her 29th week of pregnancy with no medical background, who presented with fever, headache, myalgia, and arthralgia the COVID-19 test was positive for fetal condition the obstetric ultrasound was without anomalies, fetal heart-rate (FHR) monitoring was 
normal as well, after 5 days of no clinical improvement and after consent the patient received hydroxychloroquine treatment then all symptoms disappeared after two weeks, besides her COVID-19 test was negative.

\section{DISCUSSION}

A deeply troubling matter for obstetricians during the COVID-19's pandemic is whether pregnant women would have worse outcomes compared with non-pregnant women of similar ages when infected. Studies reported that pregnant women were at a higher risk of getting infections with H1N1 influenza and SARS-CoV and were also associated with poorer clinical outcomes, like required mechanical ventilation, organ dysfunction, ICU admission and death, in comparison to reproductive aged non-pregnant women. ${ }^{4}$ Therefor a retrospective study published on the 22th April performed in The Central Hospital of Wuhan china of 28 were pregnant women with COVID-19 did not find an association between pregnancy and outcomes with patients who received antivirus treatments ribavirin and umifenovir. (included the severity of disease, virus clearance time and LOS) Similar results were also reported in a recently published case series, as in our study all patients remained stable with no pregnancy complications under hydroxychloroquine treatment. ${ }^{5,6}$

Concerning the timing of infection in our study the majority were third trimester pregnancies.

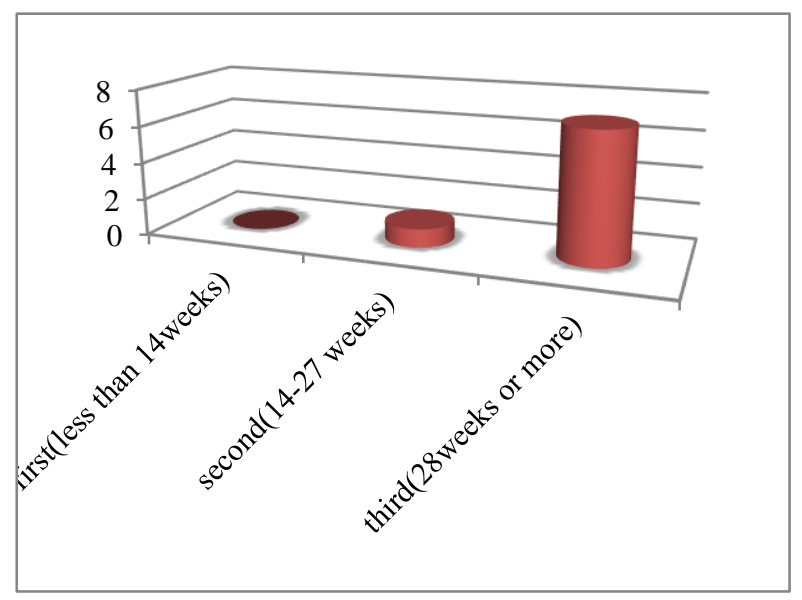

Figure 1: Pregnant women with COVID-19 by trimester of infection.

Another key concern for COVID-19 in pregnancy is whether it could be vertically transmitted from mother to child, or not, as previously reported for SARS. ${ }^{7}$ A recent review summarized 38 pregnant women and their newborns in China, and no evidence for vertical transmission were identified. ${ }^{8}$ Among the included literature, one study enrolled pregnant women with COVID-19 in the late stage of pregnancy. ${ }^{6,9}$ In our study only one woman gave birth and the baby tested negative to COVID-19.

Regarding the breastfeeding matter, there is currently insufficient evidence regarding the safety of breastfeeding and the need for mother/baby separation. If the mother is severely or critically ill, separation appears to be the best option, with attempts to express breastmilk in order to maintain milk production. ${ }^{10}$ If the patient is asymptomatic or mildly affected, breastfeeding and colocation (rooming-in) can be considered by the mother in coordination with healthcare providers. ${ }^{10}$

Concerning the duration of treatment, the hydroxychloroquine treatment seems to be more effective for COVID-19 healing, therefor there is insufficient data of using this treatment for pregnant women with COVID19.

Pregnant patients with COVID-19 received treatment after consent because there is no data on using hydroxychloroquine on pregnant women for COVID-19. As a matter of fact, this might be explained by the concern of side effects in the fetus that could be caused by certain drugs, like interferon-alpha or hydroxychloroquine. ${ }^{11,12}$ Except for the case 1 that underwent cesarean before receiving any drugs. Two pregnant patients used lopinavir as the antiviral for the treatment of COVID-19 one of then began the hydroxychloroquine for only two days, and four exclusively received hydroxychloroquine after consent. Finally, in terms of both drug use antivirus regimens and hydroxychloroquine in pregnant women, more time and studies are needed to conclude whether there is a short or long term fetal complication.

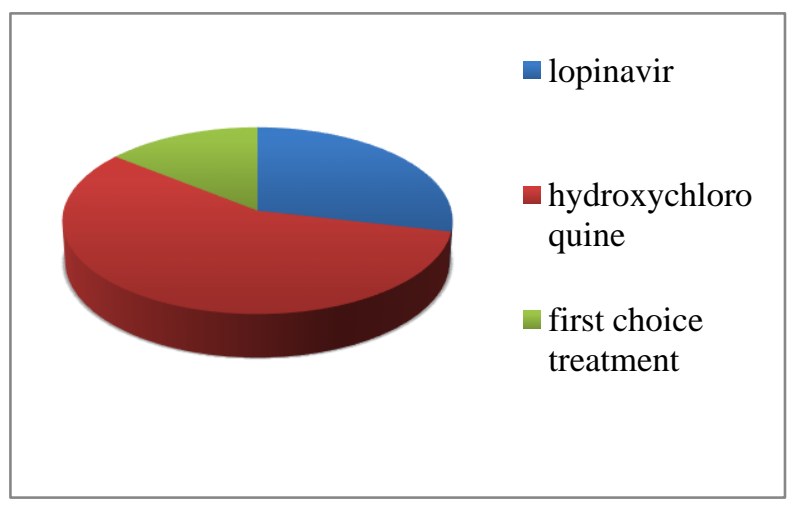

Figure 2: Pregnant women by the treatment they received in our study.

In a matter of fact there is data on using hydroxychloroquine on pregnant women for rheumatic diseases, a study preformed from June 1996 to September 2002 in Spedali Civili - Brescia hospital in Italy, 40 patients affected by rheumatic diseases were treated with HCQ during pregnancy Main outcome measures at birth were incidence of prematurity, congenital malformations and neonatal infections. Of these babies, including who 
were breast-fed, 24 were followed up during early infancy for visual function and neurodevelopmental outcome. ${ }^{13}$ And showed that there is No significant congenital malformations or neonatal infections were detected. All infants, including those who were breastfed, had normal visual function and neurodevelopmental outcome. So, Hydroxychloroquine treatment during gestation and lactation appeared to be safe. ${ }^{13}$

Another study of the impact of hydroxychloroquine treatment on pregnancy outcome in women with antiphospholipid antibodies, an observational, retrospective, single Centre cohort study. All the records of women attending the lupus and antiphospholipid pregnancy clinic London, UK between January 2008 and July 2014 were searched and has shown that HCQ Treatment was well tolerated and no side effect was reported. ${ }^{14}$ No patients suspended treatment with HCQ during the follow-up.

Several studies have shown no significant risk of congenital malformations, retinopathy or ototoxicity. ${ }^{15,16}$ Taken all above together, the risk of HCQ-related side effects seems negligible when exposure is less than 12 months, making HCQ a suitably low- risk drug to trial in pregnancy. Its use during pregnancy is now recommended in the EULAR guidelines for SLE management. ${ }^{17}$

There are several limitations in our study. First, the majority of the included patients were presented as mild to moderate, which limited the interpretation of the results. Although the reported sensitivity and specificity were good enough, the potential to introduce selection bias should still be taken into consideration, because the test might exclude true COVID-19 patients due to the low concentration of antibody. Moreover, some patients were still pregnant and hospitalized when these case reports were finalized.

\section{CONCLUSION}

Based on the current available studies of pregnant women with COVID-19 are similar to those of non-pregnant adults. There is no evidence that COVID-19 infected pregnant women are more likely to develop severe pregnancy outcomes or other maternal complication or even death. The maternal outcomes under hydroxychloroquine appear good in most cases for both mother and child and seemed to be safe according to several studies on its use for rheumatic diseases. In the absence of more robust data, this treatment might be best practice in countries that can't afford the expensive antiviral treatment in this outbreak especially in emergency situations.

Funding: No funding sources Conflict of interest: None declared Ethical approval: Not required

\section{REFERENCES}

1. Cucinotta D, Vanelli M. WHO Declares COVID-19 a Pandemic. Acta bio-medica: Atenei Parmensis 2020;91(1):157-60

2. Weetman AP. Immunity, thyroid function and pregnancy: molecular mechanisms. Nat Rev Endocrinol. 2010;6(6):311-8.

3. Longman RE, Johnson TR. Viral respiratory disease in pregnancy. Curr Opin Obstet Gynecol. 2007;19(2):120-5.

4. Creanga AA, Johnson TF, Graitcer SB, Hartman LK, Al-Samarrai T, Schwarz AG, et al. Severity of 2009 pandemic influenza A (H1N1) virus infection in pregnant women. Obstet Gynecol. 2010;115(4):71726.

5. Qiancheng X, Jian S, Lingling P, Lei H, Xiaogan J, Weihua L, et al. The sixth batch of Anhui medical team aiding Wuhan for COVID-19, Coronavirus disease 2019 in pregnancy. Int J Infect Diseas. 2020.

6. Chen H, Guo J, Wang C, Luo F, Yu X, Zhang W, et al. Clinical characteristics and intrauterine vertical transmission potential of COVID-19 infection in nine pregnant women: a retrospective review of medical records. Lancet. 2020;395(10226):809-15.

7. Lam CM, Wong SF, Leung TN, Chow KM, Ho LC. A case-controlled study comparing clinical course and outcomes of pregnant and non-pregnant women with severe acute respiratory syndrome. Int J Obstet Gynaecol. 2004;111(8):771-4.

8. Schwartz DA. An Analysis of 38 Pregnant Women with COVID-19, Their Newborn Infants, and Maternal-Fetal Transmission of SARS-CoV-2: Maternal Coronavirus Infections and Pregnancy Outcomes. Arch Pathol Lab Med. 2020.

9. Zhu H, Wang L, Fang C, Peng S, Zhang L, Chang G, et al. Clinical analysis of 10 neonates born to mothers with 2019-nCoV pneumonia. Transl Pediatr. 2020;9:51-60.

10. Poon LC, Yang H, Dumont S, et al. ISUOG Interim Guidance on coronavirus disease 2019 (COVID-19) during pregnancy and puerperium: information for healthcare professionals - an update. Ultrasound Obstet Gynecol. 2020;55(6):848-62.

11. Hiratsuka M, Minakami H, Koshizuka S, Sato I. Administration of interferon-alpha during pregnancy: effects on fetus. J Perinatal Medic. 2000;28(5):372-6.

12. Liang $\mathrm{H}$, Acharya $\mathrm{G}$. Novel corona virus disease (COVID-19) in pregnancy: What clinical recommendations to follow? Acta obstetricia et Gynecologica Scandinavica. 2020;99(4):439-42.

13. Motta M, Tincani A, Faden D, Zinzini E, Lojacono A, Marchesi A, Frassi M, Biasini C, Zatti S, Chirico G. Follow-up of infants exposed to hydroxychloroquine given to mothers during pregnancy and lactation. J Perinatol. 2005;25(2):869.

14. Sciascia S, Hunt BJ, Talavera-Garcia E, Lliso G, Khamashta MA, Cuadrado MJ. The impact of hydroxychloroquine treatment on pregnancy 
outcome in women with antiphospholipid antibodies. Am J Obstet Gynecol. 2016;214(2):273.e1-8.

15. Osadchy A, Ratnapalan T, Koren G. Ocular toxicity in children exposed in utero to antimalarial drugs: review of the literature. J Rheumat. 2011;38:2504-8.

16. Costedoat-Chalumeau N, Amoura Z, Duhaut P. Safety of hydroxychloroquine in pregnant patients with connective tissue diseases: a study of one hundred thirty-three cases compared with a control group. Arthritis Rheum. 2003;48:3207-11.
17. Bertsias G, Ioannidis JP, Boletis J. EULAR recommendations for the management of systemic lupus erythematosus. Report of a Task Force of the EULAR Standing Committee for International Clinical Studies Including Therapeutics. Ann Rheum Dis. 2008;67:195-205.

Cite this article as: Aouali $\mathrm{K}$, Moustaid $\mathrm{H}$,

Benkirane S. COVID-19 and pregnancy: the fruitful experience of the university hospital of Tangier Morocco with hydroxychloroquine and anti-retroviral treatment. Int J Reprod Contracept Obstet Gynecol 2021;10:1143-7. 